\title{
The Boltzmann Equation: Global Existence for a Rare Gas in an Infinite Vacuum*
}

\author{
Reinhard Illner ${ }^{1}$ and Marvin Shinbrot ${ }^{2}$ \\ 1 University of Kaiserslautern, D-6570 Kaiserslautern, Federal Republic of Germany \\ 2 Department of Mathematics, University of Victoria, B.C., V8W 2Y2, Canada
}

\begin{abstract}
Solutions of the Boltzmann equation are proved to exist, globally in time, under conditions that include the case of a finite volume of gas in an infinite vacuum when the mean free path of the gas is large enough. It is also proved, as might be expected in this case, that the density of the gas at each point in space goes to zero as time goes to infinity.
\end{abstract}

\section{Introduction}

Here, we prove that the Boltzmann equation has a unique solution, global in time, in all space. The conditions are a) that the initial data go to zero fast enough at infinity, and b) that the mean free path is large enough. As a special case, it is illuminating to think of a finite volume of gas released into an infinite vacuum. In this paradigmatic case, our results give that the corresponding solution of the Boltzmann equation exists globally if the gas is rare enough.

The paradigm shows that infinity should be absorbing, and so it is. Under conditions a) and b), we prove in Sect. 5 that all molecules are eventually swept out of any finite domain. Thus equilibrium is always trivial. The fact that infinity is absorbing shows that our results are related to earlier work of Babovsky [1], who proved global existence in a bounded domain when the mean free path is large, assuming that the boundary of the domain is absorbing, that is, that molecules simply disappear when they reach the boundary. Our result is, perhaps, physically more interesting since particles do not literally vanish in a finite time.

To arrive at our results, we use the method of Kaniel and Shinbrot [4], which requires a global upper bound for the solution. To find this, the basic idea is that, under conditions a) and b), the dominant process in the gas should be free flow, the molecular interactions playing a secondary role. This suggests looking for an estimate

$$
f(t, x, \xi) \leqq \tilde{f}(x-\xi t, \xi),
$$

where $f$ is the solution of the Boltzmann equation and $\tilde{f}$, which depends on $t$ only through the combination $x-\xi$ t, describes a free flow. Estimates like (1.1) were introduced by Tartar [5] for certain discrete velocity models of the Boltzmann

* Research supported by the Natural Science and Engineering Research Council Canada under Grant No. A-8560 
equation in one space dimension. Illner [3] later applied these estimates to the onedimensional Broadwell model. Recently, Hamdache [2] applied inequalities like (1.1) to a class of discrete velocity models in three space dimensions.

We carry out the argument for hard-sphere molecules. Although it is probably possible to extend the results to other kinds of molecules with a finite cut-off, we make no attempt to do so.

The conditions a) and b) seem to be essential for our approach. This is natural, since we require the flow to be dominated by free streaming. On the other hand, an interesting question is whether at least the condition on the mean free path can be omitted. Since the Boltzmann equation is derived under the hypothesis that the gas be rare, the condition may really be essential, but we conjecture that it is not.

\section{Preliminaries}

In what follows, $t$ is the time and $(x, \xi)$ the position and velocity of a molecule. Given any function defined on $[0, T) \times \mathbb{R}^{3} \times \mathbb{R}^{3}$, we always write $f^{\natural}(t, x, \xi)$ $=f(t, x+\xi t, \xi)$. This notation can be used to write the Boltzmann equation in the so-called "mild" form

$$
\frac{d}{d t} f^{\natural}(t, x, \xi)=C^{\natural}(f, f)(t, x, \xi),
$$

when there are no spatial boundaries. We look for a solution of (2.1) satisfying

$$
f(0, x, \xi)=\phi(x, \xi),
$$

where $\phi$ is given. When the molecules are hard spheres, the operator $C$ in (2.1) takes the form

$$
\begin{aligned}
C(f, f)(t, x, \xi) & =\mathscr{A} \int_{\mathbb{R}^{3}} \int_{S_{+}^{2}} \omega \cdot(\xi-\eta)\left[f(t, x, \xi) f\left(t, x, \eta^{\prime}\right)-f(t, x, \xi) f(t, x, \eta)\right] d \omega d \eta \\
& =Q(f, f)(t, x, \xi)-P(f, f)(t, x, \xi),
\end{aligned}
$$

say. Notice that $P(f, f)=f R(f)$, where

$$
\begin{aligned}
R(f)(t, x, \xi) & =\mathscr{A} \int_{\mathbb{R}^{3}} \int_{S_{\mp}^{2}} \omega \cdot(\xi-\eta) f(t, x, \eta) d \omega d \eta \\
& =\pi \mathscr{A} \int_{\mathbb{R}_{3}}|\xi-\eta| f(t, x, \eta) d \eta .
\end{aligned}
$$

$\mathscr{A}$ is a constant, proportional to the total area of the spheres; $1 / \mathscr{A}$ is a measure of the mean free path. Also

$$
S_{+}^{2}=\left\{\omega \in \mathbb{R}^{3}:|\omega|=1, \omega \cdot(\xi-\eta)>0\right\},
$$

and

$$
\xi^{\prime}=\xi-[\omega \cdot(\xi-\eta)] \omega, \quad \eta^{\prime}=\eta+[\omega \cdot(\xi-\eta)] \omega .
$$

The transformation $\mathscr{J}:(\xi, \omega, \eta) \rightarrow\left(\xi^{\prime}-\omega, \eta\right)$ is known as the collision transformation. It is an involution, so that $\mathscr{J}^{2}$ is the identity. Other well-known properties of 
$\mathscr{J}$ are conservation of momentum and energy. Since we need these properties, we record them here:

$$
\xi^{\prime}+\eta^{\prime}=\xi+\eta, \quad\left|\xi^{\prime}\right|^{2}+\left|\eta^{\prime}\right|^{2}=|\xi|^{2}+|\eta|^{2} .
$$

All our considerations take place in spaces $S_{\beta}, \beta>0$, consisting of the completion of the continuous functions of compact support with respect to the norm

$$
\|f\|_{\beta}=\int_{\mathbb{R}^{3}} \max _{x}\left|e^{\beta|x|^{2}} f(x, \xi)\right| d \xi .
$$

If $f$ and $g$ lie in $S_{\beta}$, we write $f \geqq g$, if $f(x, \xi) \geqq g(x, \xi)$ a.e. Then, we denote by $S_{\beta}^{+}$the set of all $f \in S_{\beta}$ satisfying $f \geqq 0$. With this notation, we can say what is meant by condition a) of the introduction. It is that the initial data lie in $S_{\beta}^{+}$for some $\beta>0$.

We need two other norms besides (2.7), referring to functions defined only in $\mathbb{R}^{3}$. They are

$$
\|f\|_{0}=\int_{\mathbb{R}^{3}}|f(\xi)| d \xi \quad \text { and } \quad\|f\|_{1}=\int_{\mathbb{R}^{3}}|\xi||f(\xi)| d \xi .
$$

Clearly, the spaces defined by these norms are $L^{1}$-spaces with respect to the measures $d \xi$ and $|\xi| d \xi$. We denote these spaces by $L_{0}^{1}$ and $L_{1}^{1}$, respectively.

We solve (2.1-2) using the method derived by Kaniel and Shinbrot in [4]. Since the context here is different, and also for the sake of completeness, we review the method now. Let $0<T \leqq \infty$. Suppose we have two functions $u_{0}$ and $l_{0}$ such that $u_{0}^{\natural}$ and $l_{0}^{\natural}$ map $[0, T)$ into $S_{\beta}^{+}$, and such that $l_{0}(t) \leqq u_{0}(t), 0 \leqq t<T$. Then, we define recursively two sequences $\left\{l_{k}\right\}$ and $\left\{u_{k}\right\}$ as solutions of the equations

$$
\left.\begin{array}{c}
\frac{d}{d t} l_{k+1}^{\natural}+l_{k+1}^{\natural} R^{\natural}\left(u_{k}\right)=Q^{\natural}\left(l_{k}, l_{k}\right), \\
\frac{d}{d t} u_{k+1}^{\natural}+u_{k+1}^{\natural} R^{\natural}\left(l_{k}\right)=Q^{\natural}\left(u_{k}, u_{k}\right), \\
l_{k+1}(0)=\phi=u_{k+1}(0),
\end{array}\right\}
$$

for $k=0,1, \ldots$. Suppose that

$$
u_{0}^{\natural} R^{\natural}\left(u_{0}\right), \quad Q^{\natural}\left(u_{0}, u_{0}\right) \in L_{\text {loc }}^{1}\left(0, T ; S_{\beta}^{+}\right) .
$$

Then, (2.9) has a unique solution when $k=0$. Notice that $(2.9 \mathrm{a}, \mathrm{b})$ are linear, ordinary differential equations. Accordingly, $l_{k}$ and $u_{k}$ exist when $l_{k-1}$ and $u_{k-1}$ do. Also, using the monotonicity properties of such equations as well as the monotonicity of $R$ and $Q$ in their arguments, it is easy to show that if $l_{k-1}(t) \leqq l_{k}(t)$ $\leqq u_{k}(t) \leqq u_{k-1}(t)$ for $0 \leqq t<T$, then $l_{k}(t) \leqq l_{k+1}(t) \leqq u_{k+1}(t) \leqq u_{k}(t)$ in the same interval. Thus, it follows that all equations (2.9) have unique solutions $l_{k}^{\natural}, u_{k}^{\natural} \in C^{0}\left(0, T ; S_{\beta}^{+}\right)$with $\left\{l_{k}(t)\right\}$ increasing and $\left\{u_{k}(t)\right\}$ decreasing if

$$
0 \leqq l_{0}(t) \leqq l_{1}(t) \leqq u_{1}(t) \leqq u_{0}(t) \text { for } 0 \leqq t<T .
$$

Expression (2.11) is called the beginning condition in [4].

Suppose the beginning condition is satisfied with some $u_{0}^{\natural}:[0, T) \rightarrow S_{\beta}^{+}$ satisfying (2.10). Then there are functions $l$ and $u$ such that $l_{k}(t) \uparrow l(t)$ and $u_{k}(t) \downarrow u(t)$ 
for all $t, 0 \leqq t<T$, since both sequences are surely bounded below by $l_{0}(t)$ and above by $u_{0}(t)$. Also,

$$
l(t) \leqq u(t), \quad 0 \leqq t<T .
$$

Now, integrate (2.9), send $k$ to infinity, and use the dominated convergence theorem. The result is

$$
\begin{aligned}
& l^{\natural}(t)+\int_{0}^{t} l^{\natural} R^{\natural}(u)(\tau) d \tau=\phi+\int_{0}^{t} Q^{\natural}(l, l)(\tau) d \tau, \\
& u^{\natural}(t)+\int_{0}^{t} u^{\natural} R^{\natural}(l)(\tau) d \tau=\phi+\int_{0}^{t} Q^{\natural}(u, u)(\tau) d \tau .
\end{aligned}
$$

Thus, $l^{\natural}$ and $u^{\natural}$ are in $C^{1}\left(0, T ; S_{\beta}^{+}\right)$. Differentiating, we obtain

$$
\left.\begin{array}{c}
\frac{d}{d t} l^{\natural}+l^{\natural} R^{\natural}(u)=Q^{\natural}(l, l), \\
\frac{d}{d t} u^{\natural}+u^{\natural} R^{\natural}(l)=Q^{\natural}(u, u), \\
l(0)=\phi=u(0) .
\end{array}\right\}
$$

If we can show

$$
u=l,
$$

then $f=u=l$ is a mild solution of the Boltzmann equation on $[0, T)$. We call (2.13) the separated Boltzmann system.

Now, take $l_{0}=0$ and any $u_{0}^{\natural}:[0, T) \rightarrow S_{\beta}^{+}$satisfying (2.10). Equations (2.9) with $k=0$ give easily $0=l_{0}(t) \leqq l_{1}(t) \leqq u_{1}(t)$. Thus, without further hypotheses, the beginning condition reduces to

$$
u_{1}(t) \leqq u_{0}(t) .
$$

We summarize: To solve the Boltzmann equation in an interval $[0, T)$, $0<T \leqq \infty$, we have only to find a $u_{0}$ satisfying (2.10) such that (2.15) holds on $[0, T)$, and then to verify (2.14). Precisely, we have

Lemma 1. Let $\phi \in S_{\beta}^{+}$and $l_{0}=0$. Suppose there is a function $u_{0}^{\natural}:[0, T) \rightarrow S_{\beta}^{+}$such that (2.10) holds. If, in addition, the beginning condition (2.15) is satisfied on $[0, T)$, then the separated Boltzmann system (2.13) has a solution $(l, u) \in C^{1}\left(0, T ; S_{\beta}^{+}\right)$ $\times C^{1}\left(0, T ; S_{\beta}^{+}\right)$.

\section{The Beginning Condition}

In this section, we show that the beginning condition (2.15) can be satisfied globally $(T=\infty)$ when condition b), that the mean free path is large, is satisfied. First, we have to say what we mean by a large mean free path. Let $\phi \in S_{\beta}^{+}$. Then, the function

$$
\psi(\xi)=\sup _{x} e^{\beta|x|^{2}} \phi(x, \xi)
$$


is in $L_{0}^{1}$. In the following we assume that

$$
8 \pi \mathscr{A}\|\psi\|_{0}<\sqrt{\frac{\beta}{\pi}}
$$

Since (3.2) gives an upper bound on $\mathscr{A}$, it may be interpreted as saying that the mean free path is large.

Now, when $l_{0}=0,(2.9 \mathrm{~b}, \mathrm{c})$ give

$$
u_{1}^{\natural}(t)=\phi+\int_{0}^{t} Q^{\natural}\left(u_{0}, u_{0}\right) d \tau
$$

or, what is the same thing,

$$
\begin{aligned}
u_{1}(t, x+\xi t, \eta)= & \phi(x, \xi)+\mathscr{A} \int_{0}^{t} \int_{\mathbb{R}^{3}} \int_{S_{+}^{2}} \omega \cdot(\xi-\eta) u_{0}\left(\tau, x+\xi \tau, \xi^{\prime}\right) \\
& \cdot u_{0}\left(\tau, x+\xi \tau, \eta^{\prime}\right) d \eta d \omega d \tau
\end{aligned}
$$

We now suppose, striving for an estimate (1.1), that $u_{0}$ has the form $u_{0}(t, x, \xi)$ $=v(x-\xi t, \xi)$. In this case, it follows from (3.3) that the beginning condition (2.16) is satisfied if

$$
\phi(x, \xi)+\mathscr{A} \int_{0}^{t} \int_{\mathbb{R}^{3}} \int_{S^{2}} \omega \cdot(\xi-\eta) v\left(x+\tau\left(\xi-\xi^{\prime}\right), \xi^{\prime}\right) v\left(x+\tau\left(\xi-\eta^{\prime}\right), \eta^{\prime}\right) d \omega d \eta d \tau \leqq v(x, \xi) .
$$

We note that

$$
\begin{aligned}
\mid x & +\left.\tau\left(\xi-\xi^{\prime}\right)\right|^{2}+\left|x+\tau\left(\xi-\eta^{\prime}\right)\right|^{2} \\
& =2|x|^{2}+2 \tau x \cdot\left(2 \xi-\xi^{\prime}-\eta^{\prime}\right)+\tau^{2}\left(2|x|^{2}-2 \xi\left(\xi^{\prime}+\eta^{\prime}\right)+\left|\xi^{\prime}\right|^{2}+\left|\eta^{\prime}\right|^{2}\right) \\
& =|x|^{2}+|x+\tau(\xi-\eta)|^{2}
\end{aligned}
$$

by (2.6). Thus, if we set

$$
u_{0}(t, x, \xi)=v(x-\xi t, \xi)=e^{-\beta|x-\xi t|^{2}} w(\xi),
$$

(3.4) is satisfied if

$$
\psi(\xi)+\mathscr{A} \int_{0}^{t} \int_{\mathbb{R}^{3}} \int_{S_{+}^{2}} \omega \cdot(\xi-\eta) e^{-\beta|x+\tau(\xi-\eta)|^{2}} w\left(\xi^{\prime}\right) w\left(\eta^{\prime}\right) d \eta d \omega d \tau \leqq w(\xi) ;
$$

here $\psi$ is defined in (3.1).

We are looking for a non-negative solution of (3.7). When $w \geqq 0$, on the other hand, (3.7) is true for all $t \geqq 0$ when it is true for $t=\infty$. Also,

$$
\int_{0}^{\infty} e^{-\beta|x+\tau(\xi-\eta)|^{2}} d \tau=e^{-\beta|x|^{2} \sin ^{2} \theta} \int_{0}^{\infty} e^{-\beta(\tau|\xi-\eta|+|x| \cos \theta)^{2}} d \tau,
$$


where $\theta$ is the angle between $x$ and $\xi-\eta$. Thus, we have

$$
\begin{aligned}
\int_{0}^{\infty} e^{-\beta|x+\tau(\xi-\eta)|^{2}} d \tau & \leqq \int_{0}^{\infty} e^{-\beta(\tau|\xi-\eta|+|x| \cos \theta)^{2}} d \tau \\
& \leqq \int_{-\infty}^{\infty} e^{-\beta \tau^{2}(\xi-\eta)^{2}} d \tau \\
& =\frac{1}{|\xi-\eta|} \sqrt{\frac{\pi}{\beta}}
\end{aligned}
$$

Since $0 \leqq \omega \cdot(\xi-\eta) \leqq|\xi-\eta|,(3.7)$ holds if

$$
\psi(\xi)+\mathscr{A} \cdot \sqrt{\frac{\pi}{\beta}} \int_{\mathbb{R}^{3}} \int_{S_{+}^{2}} w\left(\xi^{\prime}\right) w\left(\eta^{\prime}\right) d \omega d \eta=w(\xi) .
$$

Thus, the beginning condition is satisfied by the function (3.6) when $w$ satisfies the integral equation (3.8). We show that this is the case when (3.2) is satisfied.

Define an integral operator $W: L^{1}\left(\mathbb{R}^{3}\right) \rightarrow L^{1}\left(\mathbb{R}^{3}\right)$ by

$$
W(w)(\xi)=\psi(\xi)+\varepsilon \int_{\mathbb{R}^{3}} \int_{S_{+}^{2}} w\left(\xi^{\prime}\right) w\left(\eta^{\prime}\right) d \omega d \eta
$$

where $\varepsilon=\mathscr{A} \sqrt{\frac{\pi}{\beta}}$. W is well-defined because of the involution property of $\mathscr{J}$ and Fubini's theorem.

Let $w \geqq 0$ and integrate (3.9). The result is

$$
\begin{aligned}
\|W(w)\|_{0} & =\|\psi\|_{0}+\varepsilon \int_{\mathbb{R}^{3}} \int_{\mathbb{R}^{3}} \int_{S_{+}^{2}} w\left(\xi^{\prime}\right) w\left(\eta^{\prime}\right) d \omega d \eta d \xi \\
& =\|\psi\|_{0}+\varepsilon \int_{\mathbb{R}^{3}} \int_{\mathbb{R}^{3}} \int_{S_{+}^{2}} w(\xi) w(\eta) d \omega d \eta d \xi \\
& =\|\psi\|_{0}+2 \pi \varepsilon\|w\|_{0}^{2} .
\end{aligned}
$$

Also, if $w_{1}, w_{2}, \geqq 0$, a similar calculation shows that

$$
\left\|W\left(w_{1}\right)-W\left(w_{2}\right)\right\|_{0} \leqq 2 \pi \varepsilon\left(\left\|w_{1}\right\|_{0}+\left\|w_{2}\right\|_{0}\right)\left\|w_{1}-w_{2}\right\|_{0} .
$$

Equation (3.10) shows that $W$ maps the set of non-negative functions in the ball of radius $R_{0}$ into itself if

$$
\|\psi\|_{0}+2 \pi \varepsilon R_{0}^{2} \leqq R_{0}
$$

while (3.11) shows that $W$ is a contraction on this set if

$$
4 \pi \varepsilon R_{0}<1 .
$$

Inequalities (3.12) and (3.13) can both be satisfied by choosing $R_{0}$ so as to satisfy

$$
1-\sqrt{1-8 \pi \varepsilon\|\psi\|_{0}} \leqq 4 \pi \varepsilon R_{0}<1,
$$

and this is possible when $8 \pi \varepsilon\|\psi\|_{0}<1$. By the definition of $\varepsilon$, this is the case when (3.2) holds. Thus, (3.8) has a non-negative solution and the beginning condition can be satisfied globally when we have (3.2). 
Since $W$ is a contraction, the solution of (3.8) is of course given by $w=\lim _{k \rightarrow \infty} W^{k}(\psi)$. If $\left\|W^{k}(\psi)\right\|_{0} \leqq 2\|\psi\|_{0}$ (which is true when $k=0$ ), it follows from (3.10) that $\left\|W^{k+1}(\psi)\right\|_{0} \leqq\|\psi\|_{0}+8 \pi \varepsilon\|\psi\|_{0}^{2} \leqq 2\|\psi\|_{0}$, by (3.2). Thus,

$$
\left\|W^{k}(\psi)\right\|_{0} \leqq\|w\|_{0} \leqq 2\|\psi\|_{0} \text { for all } k .
$$

Suppose next that $\psi \in L_{0}^{1} \cap L_{1}^{1}$. If $w \in L_{1}^{1}$, we have, from (3.9),

$$
\begin{aligned}
\|W(w)\|_{1} & =\|\psi\|_{1}+\varepsilon \int_{\mathbb{R}^{3}} \int_{\mathbb{R}^{3}} \int_{S^{2}}|\xi| w\left(\xi^{\prime}\right) w\left(\eta^{\prime}\right) d \omega d \eta d \xi \\
& =\|\psi\|_{1}+\frac{\varepsilon}{2} \int_{\mathbb{R}^{3}} \int_{\mathbb{R}^{3}} \int_{S_{\mp}^{2}}(|\xi|+|\eta|) w\left(\xi^{\prime}\right) w\left(\eta^{\prime}\right) d \omega d \eta d \xi \\
& =\|\psi\|_{1}+\frac{\varepsilon}{2} \int_{\mathbb{R}^{3}} \int_{\mathbb{R}^{3}} \int_{S^{2}}\left(\left|\xi^{\prime}\right|+\left|\eta^{\prime}\right|\right) w(\xi) w(\eta) d \omega d \eta d \xi .
\end{aligned}
$$

However, $\left|\xi^{\prime}\right|+\left|\eta^{\prime}\right| \leqq \sqrt{2} \sqrt{\left|\xi^{\prime}\right|^{2}+\left|\eta^{\prime}\right|^{2}}=\sqrt{2} \sqrt{|\xi|^{2}+|\eta|^{2}} \leqq \sqrt{2}(|\xi|+|\eta|)$. Using this inequality in (3.15), we find

$$
\|W(w)\|_{1} \leqq\|\psi\|_{1}+2 \pi \varepsilon \sqrt{2}\|w\|_{0}\|w\|_{1} .
$$

Thus, $W$ maps $L_{0}^{1} \cap L_{1}^{1}$ into itself. Applying (3.16) to $W^{k}(\psi)$, we get

$$
\begin{aligned}
\left\|W^{k+1}(\psi)\right\|_{1} & \leqq\|\psi\|_{1}+2 \pi \varepsilon \sqrt{2}\left\|W^{k}(\psi)\right\|_{0}\left\|W^{k}(\psi)\right\|_{1} \\
& \leqq\|\psi\|_{1}+4 \pi \varepsilon \sqrt{2}\|\psi\|_{0}\left\|W^{k}(\psi)\right\|_{1} \\
& \leqq\|\psi\|_{1}+\frac{1}{\sqrt{2}}\left\|W^{k}(\psi)\right\|_{1},
\end{aligned}
$$

where (3.14) and (3.2) have been used. An induction now gives $\left\|W^{k}(\psi)\right\|_{1}$ $\leqq\|\psi\|_{1} \frac{\sqrt{2}}{\sqrt{2}-1}$. Thus, if $\psi \in L_{0}^{1} \cap L_{1}^{1}$, the solution of (3.8) lies in the same space. We need the fact just proved to show that Lemma 1 may be applied, for we have to show that $Q\left(u_{0}, u_{0}\right)$ and $R\left(u_{0}\right)$ satisfy the conditions of the lemma. We proved in the course of deriving $(3.8)$ that $Q^{\natural}\left(u_{0}, u_{0}\right) \in L^{1}\left(0, \infty ; S_{\beta}^{+}\right)$, and in a similar way one sees that $u_{0}^{\natural} R^{\natural}\left(u_{0}\right) \in L^{1}\left(0, \infty ; S_{\beta}^{+}\right)$.

Thus, we have

Lemma 2. Let $\phi \in S_{\beta}^{+}$and define $\psi$ by (3.1). If (3.2) holds and if $\psi \in L_{0}^{1} \cap L_{1}^{1}$, then the separated Boltzmann system (2.13) has a global solution $(l, u)$ with $\left(l^{\natural}, u^{\natural}\right):[0, \infty)$ $\rightarrow S_{\beta}^{+} \times S_{\beta}^{+}$.

\section{The Boltzmann Equation}

To solve the Boltzmann equation itself, it remains to show that $l=u,(l, u)$ being the solution of the separated Boltzmann system (2.13). We do this by estimating $u-l$ in a suitable norm, namely, that defined by

$$
\mathbf{\square} \mathbf{\square}_{\beta}=\int_{\mathbb{R}^{3}} \sup _{t, x} e^{\beta|x|^{2}}\left|f^{\natural}(t, x, \xi)\right| d \xi .
$$


We denote by $C_{\beta}$ the set of all $f$ such that $f^{\natural} \in C^{0}\left(0, \infty ; S_{\beta}\right)$ and $\square f \mathbf{m}_{\beta}<\infty$, and by $C_{\beta}^{+}$the non-negative functions in $C_{\beta}$. Because $0 \leqq l(t) \leqq u(t) \leqq u_{0}(t)$, and because of (3.6), $l$ and $u$ lie in $C_{\beta}$.

Subtract (2.13a) from (2.13b) and integrate the result. Because of (2.13c), we find

$$
\left(u^{\natural}-l^{\natural}\right)(t)=\int_{0}^{t}\left[Q^{\natural}(u, u-l)+Q^{\natural}(u-l, l)+u^{\natural} R^{\natural}(u-l)+\left(u^{\natural}-l^{\natural}\right) R^{\natural}(l)\right](\tau) d \tau .
$$

Thus,

$$
\begin{gathered}
\mathbf{\mathbf { m } u - l \mathbf { m } _ { \beta } \leqq} \sup _{x} e^{\beta|x|^{2}} \int_{0}^{\infty}\left[Q^{\natural}(u, u-l)+Q^{\natural}(u-l, l)+u^{\natural} R^{\natural}(u-l)\right. \\
\left.+\left(u^{\natural}-l^{\natural}\right) R^{\natural}(l)\right](\tau, x, \xi) d \tau d \xi .
\end{gathered}
$$

We begin by estimating the first term on the right of (4.1). It is

$$
\begin{gathered}
\mathscr{A} \int_{\mathbb{R}^{3}} \sup _{x} e^{\beta|x|^{2}} \int_{0}^{\infty} \int_{\mathbb{R}^{3}} \int_{S_{+}^{2}} \omega \cdot(\xi-\eta) u\left(\tau, x+\tau \xi, \xi^{\prime}\right)(u-l)\left(\tau, x+\tau \xi, \eta^{\prime}\right) d \omega d \eta d \tau d \xi \\
=\mathscr{A} \int_{\mathbb{R}^{3}} \sup _{x} e^{\beta|x|^{2}} \int_{0}^{\infty} \int_{\mathbb{R}^{3}} \int_{S_{+}^{2}} \omega \cdot(\xi-\eta)\left[e^{\beta|z|^{2}} u\left(\tau, z+\tau \xi^{\prime}, \xi^{\prime}\right)\right] \\
\cdot\left[e^{\beta|y|^{2}}(u-l)\left(\tau, y+\tau \eta^{\prime}, \eta^{\prime}\right)\right] e^{-\beta\left(|z|^{2}+|y|^{2}\right)} d \omega d \eta d \tau d \xi,
\end{gathered}
$$

where we have written $z=x+\tau\left(\xi-\xi^{\prime}\right), y=x+\tau\left(\xi-\eta^{\prime}\right)$. But, according to (3.5), $|z|^{2}+|y|^{2}=|x|^{2}+|x+\tau(\xi-\eta)|^{2}$. Thus, the first term on the right of (4.1) is surely bounded by

$$
\begin{aligned}
\mathscr{A} & \int_{\mathbb{R}^{3}} \sup _{x} \int_{0}^{\infty} \int_{\mathbb{R}^{3}} \int_{S^{2}} \omega \cdot(\xi-\eta) \sup _{z}\left[e^{\beta|z|^{2}} u^{\natural}(\tau, z, \xi)\right] \\
& \cdot \sup _{y}\left[e^{\beta|y|^{2}}\left(u^{\natural}-l^{\natural}\right)(\tau, y, \eta)\right] e^{-\beta|x+\tau(\xi-\eta)|^{2}} d \omega d \eta d \tau d \xi \\
= & \mathscr{A} \int_{\mathbb{R}^{3}} \sup _{x} \int_{0}^{\infty} \int_{\mathbb{R}^{3}} \int_{S^{2}} \omega \cdot(\xi-\eta) \sup _{z}\left[e^{\beta|z|^{2}} u^{\natural}(\tau, z, \xi)\right] \\
& \cdot \sup _{y}\left[e^{\left.\beta|y|\right|^{2}}\left(u^{\natural}-l^{\natural}\right)(\tau, y, \eta)\right] e^{-\beta|x+\tau(\xi-\eta)|^{2}} d \omega d \eta d \tau d \xi \\
\leqq & \mathscr{A} \int_{\mathbb{R}^{3}} \int_{\mathbb{R}^{3}} \int_{S_{+}^{2}} \sup _{\sigma, z}\left[e^{\beta|z|^{2}} u^{\natural}(\sigma, z, \xi)\right] \sup _{\varrho, y}\left[e^{\beta|y|^{2}}\left(u^{\natural}-l^{\natural}\right)(\varrho, y, \eta)\right] \omega \\
& \cdot(\xi-\eta) \sup _{x} \int_{0}^{\infty} e^{-\beta|x+\tau(\xi-\eta)|^{2}} d \tau d \omega d \eta d \xi .
\end{aligned}
$$

Estimating the time integral as in Sect. 3, we find that (4.2) is bounded by $2 \pi \mathscr{A} \sqrt{(\pi / \beta)} \boldsymbol{\sim} u \mathbf{m}_{\beta} \boldsymbol{m} u-l \mathbf{m}_{\beta}$.

It is a straightforward matter to verify that the other three terms on the right of (4.1) have the same bound. Hence,

$$
\mathbf{\square} u-l \mathbf{\varpi}_{\beta} \leqq 8 \pi \mathscr{A} \sqrt{\frac{\pi}{\beta}} \mathbf{\mathbf { m }} u \mathbf{\varpi}_{\beta} \mathbf{\mathbf { m }} u-l \mathbf{\varpi}_{\beta} .
$$

However, $\square u \mathbf{m}_{\beta} \leqq u_{0} \mathbf{m}_{\beta}=\|w\|_{0} \leqq 2\|\psi\|_{0}$ by (3.14), and so

$$
\mathbf{\square} u-l \mathbf{m}_{\beta} \leqq 16 \pi \mathscr{A} \sqrt{\frac{\pi}{\beta}}\|\psi\|_{0} \boldsymbol{\square} u-l \mathbf{m}_{\beta} .
$$


If we now strengthen (3.2) to read

$$
q:=16 \pi \mathscr{A} \sqrt{\frac{\pi}{\beta}}\|\psi\|_{0}<1
$$

(4.3) gives $\mathbf{\square} u-l \mathbf{\square}_{\beta} \leqq q \square u-l \mathbf{\square}_{\beta}$ with $q<1$, which is impossible unless $u=l$.

We summarize our results in

Theorem 1. Let $\phi \in S_{\beta}^{+}$, and define $\psi$ by (3.1). If (4.4) holds, and if $\psi \in L_{0}^{1} \cap L_{1}^{1}$, then the initial-value problem (2.1-2) for the Boltzmann equation has a global solution $f \in C_{\beta}^{+}$. This solution is unique.

Proof. Everything but uniqueness follows from the earlier discussion in Sects. 3, 4. On the other hand, uniqueness follows exactly the proof that $u=l$ given above.

\section{Some Remarks}

Remark 5.1. In the introduction, we interpreted (4.4) as a condition that the mean free path be large. It is clear, however, that the dimensionless condition (4.4) may also be interpreted as a condition that the initial data be small.

Remark 5.2. The way we have derived the bound $u_{0}$ also shows that the monotone sequence $\left\{f_{k}\right\}$ defined by $f_{0}=0$, and

$$
\frac{d}{d t} f_{k+1}^{\natural}=Q^{\natural}\left(f_{k}, t_{k}\right), \quad f_{k+1}(0)=\phi
$$

is also bounded by $u_{0}$. Thus, the mild Boltzmann equation without the loss term,

$$
\frac{d f^{\natural}}{d t}=Q^{\natural}(f, f), \quad f(0)=\phi,
$$

also has global solution under the conditions of Theorem 1.

Remark 5.3. The fact that $f^{\natural}(t, \cdot) \in S_{\beta}^{+}$for all $t$ permits the prediction of the asymptotic behavior of the solution as $t \rightarrow \infty$. In fact, $f(t, x, \xi) \leqq u_{0}(t, x, \xi)$ $=e^{-\beta|x-\xi t|^{2}} w(\xi)$, where $w \in L_{0}^{1}$. Clearly, the form of $u_{0}$ implies that

$$
\lim _{t \rightarrow \infty} f(t, x, \xi)=0 \text { a.e. in } \mathbb{R}^{3} \times \mathbb{R}^{3} .
$$

If $w \in L_{0}^{1} \cap L^{\infty}$, we can even say more. Consider the spatial density

Clearly,

$$
\varrho(t, x):=\int_{\mathbb{R}^{3}} f(t, x, \xi) d \xi .
$$

$$
\varrho(t, x) \leqq \int_{\mathbb{R}^{3}} e^{-\beta|x-\xi t|^{2}} w(\xi) d \xi=\frac{1}{t^{3}} \int_{\mathbb{R}^{3}} e^{-\beta y^{2}} w\left(\frac{x-y}{t}\right) d y,
$$

and if $w$ is bounded it follows that

$$
|\varrho(x, t)|=0\left(\frac{1}{t^{3}}\right) \text { as } t \rightarrow \infty .
$$

Remark 5.4. One might think that the global existence proof given in Sects. 3 and 4 immediately translates into a local existence proof if $\phi \in S_{\beta}^{+}$, even if condition (4.4) is violated. Unfortunately, this is not the case. The reason is that the integral 
$\int_{0}^{t} e^{-\beta|x+s(\xi-\eta)|^{2}} d s$ can not be made small (uniformly in $\xi$ and $\eta$ ) by just choosing $t$ sufficiently small. Of course, this integral is globally bounded by $\frac{1}{|\xi-\eta|} \cdot \sqrt{\frac{\pi}{\beta}}$, but this is not sufficient for local existence. On the other hand, local existence is true if one considers more special initial data. For details, see [4].

Remark 5.5. The space $S_{\beta}$ arose in a natural way in the verification of the beginning condition, given in Sect. 3. For the proof that $u=l$, then, we had to introduce the norm given by (4.2). However, once this norm and the corresponding space are given, we can also use a contraction mapping argument to prove the global existence theorem.

Let $\phi \in S_{\beta}^{+}$, let $\psi$ be given by (3.1), and suppose that (4.4) holds. In $C_{\beta}$, let $B_{q}$ be the ball $\left\{f: 8 \pi \mathscr{A} \sqrt{\frac{\pi}{\beta}}\|f\| \leqq q\right\}$, where $\frac{1}{2}<q<1$.

Theorem 2. Suppose that $\phi \in S_{\beta}^{+}$and that (4.4) holds. Then, the initial value problem (2.1-2) has a unique global solution in $B_{q}$ when $q \in\left(\frac{1}{2}, 1\right)$.

Proof. Consider the operator $M: C_{\beta} \rightarrow C_{\beta}$ defined by \[ (M f)^{\natural}(t, x, \xi):=\phi(x, \xi)+\int_{0}^{t} C^{\natural}(f, f)(\tau, x, \xi) d \tau . \]
For $q=16 \pi \mathscr{A} \sqrt{\frac{\pi}{\beta}}\|\psi\|_{0}, M$ maps $B_{q}$ into itself and is a contraction. All the details
for this fact are already contained in the formulas (4.1) ff., and we leave the for this fact are already contained in the formulas (4.1) ff., and we leave the elaboration to the reader.

Remark 5.6. Note that the conditions in Theorem 2 are slightly less restrictive than those in Theorem 1. On the other hand, the solution given by Theorem 1 is automatically nonnegative, whereas we do not know this for the solution given by Theorem 2. We conjecture that standard methods can be applied to prove the nonnegativity also in the more general case, but make no attempt to verify this.

Acknowledgement. M. S. would like to acknowledge the hospitality and support of Harvard University and the University of Kaiserslautern during the period when this paper was written.

\section{References}

1. Babovsky, H.: Randbedingungen in der kinetischen Theorie und Lösungen der BoltzmannGleichung. Ph. D. Dissertation, Universität Kaiserslautern 1983

2. Hamdache, K.: Existence globale et comportement asymptotique pour l'equation de Boltzmann à repartition discrète de vitesses. To appear in Journal de Méc. Th. et Appl.

3. Illner, R.: The Broadwell model for initial values in $L_{+}^{1}(R)$. Commun. Math. Phys. 93, 341-353 (1984)

4. Kaniel, S., Shinbrot, M.: The Boltzmann equation. I. Uniqueness and global existence. Commun. Math. Phys. 59, 65-84 (1978)

5. Tartar, L.: Some existence theorems for semilinear hyperbolic systems in one space variable. MRC Technical Summary Report, Madison 1980

Communicated by J. L. Lebowitz

Received January 31, 1984 